\title{
Restless legs syndrome in systemic lupus erythematosus: A case-control study
}

\author{
CRISTIAN FALUP-PECURARIU ${ }^{1,2}$, ANCA ENACHE $^{2,8}$, LILIANA DUCA $^{3}$, CAMIL FOTESCU $^{2,8}$, \\ OANA FALUP-PECURARIU ${ }^{2,4}$, VLAD MONESCU $^{5}$, ŞTEFANIA DIACONU ${ }^{1,2}$ and CARMEN ADELLA SIRBU ${ }^{6,7}$ \\ ${ }^{1}$ Department of Neurology, County Emergency Clinic Hospital, 500365 Brasov; ${ }^{2}$ Faculty of Medicine, \\ Transilvania University, 500036 Brasov; ${ }^{3}$ Department of Immunology, County Emergency Clinic Hospital; \\ ${ }^{4}$ Department of Pediatrics, Children's Clinic Hospital, 500365 Brasov; ${ }^{5}$ Faculty of Mathematics and Informatics, \\ Transilvania University, 500036 Brasov; ${ }^{6}$ Department of Neurology, Faculty of Medicine, \\ 'Titu Maiorescu' University, 031593 Bucharest; 7 Department of Neurology, 'Carol Davila' \\ Central Military Emergency University Hospital, 010242 Bucharest, Romania
}

Received March 12, 2021; Accepted April 13, 2021

DOI: $10.3892 /$ etm.2021.10234

\begin{abstract}
Sleep disturbances in systemic lupus erythematosus (SLE) are not well understood. The restless legs syndrome (RLS) is one of the frequent occurring sleep disturbances in SLE. The aim of this study was to evaluate the prevalence of RLS and its characteristics in SLE. We evaluated, in a prospective case-control study, 26 patients with SLE and 26 patients without SLE in an age- and sex-matched control group. An RLS-positive diagnosis met International RLS Study Group (IRLSSG) criteria. We used standardized questionnaires, which included demographic data, medical history and sleep assessment. We used validated questionnaires and scales to assess sleep. There were $23 / 26$ females $(88.46 \%)$ in each group; the mean patient age in the SLE subgroup was 51.65 years, while in the control subgroup, 52.07 years (range $30-74)$. Nine (34.2\%) patients had RLS-positive criteria in the SLE group and 2 (7.69\%) of 26 in the control group. Eight out of 9 patients described RLS onset after SLE was diagnosed. In the SLE group, 8 cases were of moderate severity and 1 was considered mild. The control group had one mild and one moderate case of RLS. RLS prevalence in SLE is higher and the quality of sleep is poorer compared with the control group.
\end{abstract}

Correspondence to: Dr Oana Falup-Pecurariu, Faculty of Medicine, Transilvania University, 56 Nicolae Bălcescu Street, 500036 Brasov, Romania

E-mail: oanafp@yahoo.co.uk

Present address: ${ }^{8}$ Department of Anesthesia and Intensive Care, 'Pius Brînzeu' County Emergency Clinic Hospital Timisoara, Bulevardul Liviu Rebreanu 156, Timișoara 300723, Romania

Key words: Epworth sleepiness scale, Pittsburgh sleep quality index, restless legs syndrome, sleep disturbances, systemic lupus erythematosus

\section{Introduction}

Restless legs syndrome (RLS) includes various sensitive symptoms and is identified if following criteria are fulfilled: i) the need to move the feet; ii) the patient perceives this sensation as uncomfortable and unpleasant; iii) this sensation is relieved by movement; iv) a circadian rhythm was described, meaning that the unpleasant sensation starts at evening time, especially when the patient is in bed. The same sensation may appear during the daytime in advanced cases, especially in the periods of rest or inactivity $(1,2)$. The consensus published on 2003 by the International RLS Study Group (IRLSSG) stipulates that all the above four criteria need to be met to establish the diagnosis (1). These criteria were updated in 2014 (2) by adding an additional diagnostic criteria, requiring that no other medical or behavioral condition may explain the appearance of the symptoms mentioned above (2).

The prevalence of RLS is approximately $10 \%$ in different epidemiological studies (3-5), with a predominance of females (3). Clinically significant RLS has an estimated prevalence of approximately $2.7 \%$. Worldwide RLS is often underdiagnosed and is considered to significantly impair sleep and quality of life (4-7). According to Allen and colleagues, more than half of the RLS patients report at least one sleep impairment, for instance insomnia, sleep fragmentation or daytime sleepiness (6). RLS patients declare worse global quality of life and important impairments of mental and general physical status compared to healthy individuals or to patients with other conditions (6). There are several diseases/conditions which are suggested to increase the prevalence of RLS: Renal failure (8), kidney transplantation (9), rheumatoid arthritis (10), fibromyalgia (11), lung transplantation (12), migraine (13), ankylosing spondylitis (14) or multiple sclerosis (15). In pregnancy, the prevalence of RLS is also high, but in contrast with the aforementioned conditions, it has a good prognosis, remitting in few weeks/months after delivery (16). Yet, few studies have assessed RLS and sleep disturbances in systemic lupus erythematosus (SLE) $(17,18)$. 
Pharmacological treatment of RLS may include iron supplementation in cases of low serum ferritin levels, dopamine agonists as first-line therapeutic options and anticonvulsivants or opioides in refractory cases (10).

The aims of this study were: i) To determine the prevalence of RLS in SLE patients; ii) to evaluate the clinical phenotype of RLS in SLE patients; iii) to discover an association between RLS and sleep characteristics and iv) to assess sleep disturbances in these patients.

\section{Patients and methods}

Design. A prospective study was performed at the Departments of Immunology and Neurology of the County Emergency Clinic Hospital, Braşov, Romania, during the period June 2011-August 2013. This study enrolled 26 patients with SLE and 26 patients without SLE in an age- and sex-matched control group. No patient was previously diagnosed with RLS. The protocol for this study received acceptance from the Ethics Committee of the Transilvania University from Braşov (78/28.05.2011) and respects the ethical recommendations of the 1975 Declaration of Helsinki. Informed consent was signed by all subjects before recruitment. The study group consists of: i) consecutive patients with confirmed SLE diagnosed by an immunologist or rheumatologist; ii) patients older than 18 years of age; iii) able to sign an informed consent. The SLE patients presented with different affected organs but no central nervous system (CNS) involvement.

Exclusion criteria included: i) Uncertain SLE diagnosis; ii) medical or psychiatric comorbidities that could interfere with the diagnosis of RLS iii) inability/refusal to provide study consent.

The control group included consecutive patients with no SLE. Among the symptoms, they presented low back pain with/without radicular syndrome or different types of headache.

Assessments. We collected demographic data, medical history, weight, height [for body mass index (BMI)] sleep and RLS assessment. A RLS-positive diagnosis was based on the four standardized criteria proposed by the International RLS Study Group (IRLSSG) as mentioned in the introduction (1). The assessment work included demographic data, medical history and history of the use of medications. The patients had to choose from a list of 21 terms which described the sensory sensations in their legs, according to Allen et al (1). The questionnaire comprised also questions related to sleep characteristics: Time to go to bed, time to wake up, number of hours that the subject sleeps.

The following scales were applied to both groups: Restless Legs Syndrome Rating-IRLS (19) to assess the severity of a patient's RLS symptoms; Restless Legs Syndrome Quality of Life Instrument (20); Pittsburg Sleep Quality Index (PSQI) (21); Epworth Sleepiness Scale (22). According to the Restless Legs Syndrome Rating Scale-IRLS (19), the scores for all items vary between 0 (without signs of RLS) to 4 (very intense RLS symptoms); the maximum score being 40 . Therefore, according to the obtained scores, the categories of severity were: Mild, 1-10; moderate, 11-20); severe, 21-30; very severe, 31-40.
Statistical analysis. Statistical analysis was performed using Microsoft Excel 2010 software (Microsoft) and Matlab 2019b (MathWorks). Data are presented as the mean \pm standard deviation. For demographics and clinical assessment, we used descriptive statistics. Statistical differences between groups were analyzed using the one-way ANOVA test of variance followed by the Tukey's post hoc test or student's t-test. $\mathrm{P}<0.05$ was considered to indicate a statistically significant difference.

\section{Results}

Demographic data are presented in Table I. RLS+ represents all the patients fulfilling the RLS study group-positive criteria and RLS-were patients without RLS. The mean patient age was similar in the two groups: 51.65 years in patients with SLE and 52.07 years in patients without SLE (range 30-74). There were $9 / 26$ patients $(34.62 \%$ ) who fulfilled RLS-positive criteria (RLS+) (positive answers to all the 4 diagnostic criteria questions) in the study group and 2/26 (7.69\%) RLS+ patients in the control group $(\mathrm{P}<0.05)$. Family history was positive for 2 out of 9 patients with RLS in patients with SLE and none in the control group. We compared the characteristics of the patients in the study group with SLE and RLS (RLS+) with those from control group with RLS (RLS+). In the SLE and RLS+ group, compared with the control group with RLS (RLS+), we observed that the patients were older (mean age, $56.66 \pm 10.40$ years vs. $48.5 \pm 0.7$ years), predominantly females ( $7 / 9$ vs. $2 / 2$ ), with a higher prevalence of iron deficiency anemia $(\mathrm{P}<0.05)$.

According to our results, SLE patients reported more frequent and more intense symptoms of RLS compared to the control subgroup. RLS symptoms in the study group $(n=9)$ had as frequency: 1 day/month in 1 patient, 1-2 days/week in 1 patient, 2-3 days/week in 2 patients, 4-5 days/week in 1 patient and daily in 4 patients. In the control group, one patient with RLS had daily symptoms and one had symptoms 1-2 days/week. In the study group, all participants with RLS, except one, described that the RLS symptoms became manifest after the diagnosis of SLE. The mean interval from the diagnosis of SLE and the occurrence of RLS was $3.31 \pm 2.2$ years.

In the study group $(n=9)$, the mean IRLS score (19) had the following distribution: Mild (0-10) in 1 case, moderate (11-20) in 8 cases. There were no cases considered appropriate from inclusion in the severe (21-30) or very severe (31-40) group. For the controls there was one mild case (0-10) and one moderate case (11-20).

We compared clinical and laboratory exams of the SLE study patients with (RLS+) and without RLS (RLS-). Female representation was 7/9 (77. 77\%) in the RLS+ vs. 16/17 (94.11\%) in the RLS-group. There were 2 patients with polyneuropathy and RLS+ in the SLE group but none with polyneuropathy among RLS-.In the control group there were no polyneuropathy cases. The SLE study group patients received standard treatment, according to the guidelines in use, depending on the disease severity: Non-steroidal anti-inflammatory drugs, corticosteroids, hydroxychloroquine, immunosuppressants, biological therapies. No patient was diagnosed previously with RLS and no specific treatment was administered. 
Table I. Demographic and laboratory data: Comparison between the study group [patients with SLE, with (+) and without (-) RLS] and control group [patients without SLE, with (+) and without (-) RLS].

\begin{tabular}{|c|c|c|c|c|c|}
\hline & \multicolumn{2}{|c|}{ Study group $(n=26)$} & \multicolumn{2}{|c|}{ Control group $(n=26)$} & \multirow{2}{*}{$\frac{\text { P-value }}{0.04}$} \\
\hline & RLS+ $(n=9)$ & RLS- $(n=17)$ & RLS+ $(n=2)$ & RLS- $(n=24)$ & \\
\hline Age (years) & $56.66 \pm 10.40$ & $50.05 \pm 10.52$ & $48.5 \pm 0.7$ & $52.62 \pm 11.44$ & 0.04 \\
\hline BMI & $27.31 \pm 3.90$ & $25.79 \pm 6.19$ & $31.35 \pm 2.71$ & $28.83 \pm 4.87$ & 0.12 \\
\hline \multicolumn{6}{|l|}{ Sex } \\
\hline Male & 2 & 1 & 0 & 3 & 0.07 \\
\hline Female & 7 & 16 & 2 & 21 & 0.03 \\
\hline Iron deficiency anemia & 5 & 0 & 1 & 0 & 0.02 \\
\hline
\end{tabular}

Data are presented as means \pm standard deviation. SLE, systemic lupus erythematosus; RLS, restless legs syndrome; BMI, body mass index.

Table II. Summary of sleep characteristics: Comparison between the study group [patients with SLE, with (+) and without (-) RLS] and control group [patients without SLE, with (+) and without (-) RLS].

\begin{tabular}{|c|c|c|c|c|c|}
\hline \multirow[b]{2}{*}{ Sleep characteristics } & \multicolumn{2}{|c|}{ Study group } & \multicolumn{2}{|c|}{ Control group } & \multirow[b]{2}{*}{ P-value ${ }^{a}$} \\
\hline & RLS+ & RLS- & RLS+ & RLS- & \\
\hline Epworth Sleepiness Scale score (range) & $5.71 \pm 1.92(2-8)$ & $4.41 \pm 2.37(0-9)$ & $5.12 \pm 1.85$ & $4.95 \pm 1.96(0-9)$ & 0.09 \\
\hline PSQI score & $5.83 \pm 2.41$ & $3.24 \pm 1.42$ & $6.14 \pm 1.71$ & $2.95 \pm 2.12$ & 0.03 \\
\hline Time to go to bed (hour:min) & $22: 45 \pm 1: 00$ & $22: 00 \pm 0: 47$ & $23: 50 \pm 0: 42$ & $22: 00 \pm 0: 45$ & 0.82 \\
\hline Wake up time (hour:min) & $6.45 \pm 1.25$ & $8.00 \pm 1.20$ & $6.00 \pm 1.45$ & $7.00 \pm 1.10$ & 0.04 \\
\hline Time in bed (hour:min) & $6.5 \pm 2.1$ & $7.3 \pm 2.8$ & $6.45 \pm 1.92$ & $7.00 \pm 2.9$ & 0.90 \\
\hline Sleep duration (mean, hour) & 5.5 & 7 & 6 & 7.2 & 0.03 \\
\hline
\end{tabular}

Data are presented as mean \pm standard deviation. ${ }^{a}$ Comparison between study and control groups. SLE, systemic lupus erythematosus; RLS, restless legs syndrome; PSQI, Pittsburgh Sleep Quality Index.

Sleep characteristics are presented in Table II: Epworth Sleepiness Scale score, PSQI score, time to go to bed (hour:min), wake up time (hour:min), time in bed (hour:min), sleep duration (mean, hour). The sleep characteristics were comparatively analyzed in the study and control group. PSQI score (higher in the RLS+ subgroup, $\mathrm{P}=0.03$ ), time to wake up (earlier in the RLS+ subgroup, $\mathrm{P}=0.04$ ) and sleep duration (less in the $\mathrm{RLS}+$ subgroup, $\mathrm{P}=0.03$ ) reached statistical significance between the study and control groups.

Excessive daytime sleepiness [Epworth Sleepiness Scale score $(\mathrm{ESS})>10$ ] was not reported by any patient. ESS score of $6-10$ was reported by $69.23 \%$ vs. $46.15 \%(\mathrm{P}=0.01)$ in the two groups.

\section{Discussion}

This study shows a higher prevalence of restless legs syndrome (RLS) in systemic lupus erythematosus (SLE) patients (34.62\%) compared with controls (7.69\%). The RLS prevalence in the SLE group was higher compared with the one reported in the general population, which varies at approximately $10 \%(4,5)$. Hassan et al (17) showed that 12 of $33(36.36 \%)$ patients with SLE had RLS compared with 4 of 32 control patients (12.5\%).
Kucuk et al (18) also suggested a high prevalence of RLS in SLE patients (30.6\% vs. $4.8 \%$ ). Even if RLS prevalence appears to be higher in SLE patients, the pathological mechanisms remain unclear. Possible explanations include genetic susceptibility and the existence of antibodies against dopaminergic cells in SLE which disturb the dopaminergic functionality, therefore increasing the risk of RLS occurrence in these patients (17). Anemia is reported as a risk factor for RLS prevalence and severity (18). Iron deficiency anemia was more prevalent in our study in the SLE group. Due to our limited sample, we were unable to demonstrate an association between RLS and anemia, as shown by Kucuk et al (18).

The presence of RLS in systemic lupus erythematosus (SLE) impacts the quality of sleep; higher PSQI scores (meaning poorer sleep quality), earlier time to wake up and shorter total sleep time were reported.

There is limited available information regarding sleep disturbances in SLE patients with RLS, but considering the potential impairment of sleep quality induced by RLS, further studies (including also objective sleep assessments) are required.

There are some limitations in our study: i) Enrollment of a population visiting the hospital clinic could have added more 
comorbidities and thus more potential factors involved in the leg sensations; and ii) electromyography and nerve conduction studies were not performed in any patient. Hence, the two patients had clinical polyneuropathy.

In conclusion, the present study showed that SLE patients reported a higher prevalence of RLS, most of them being females. An association between RLS and iron-deficiency anemia was identified in the study group. The majority of our patients reported mild or moderate RLS symptoms, occurring in 58\% of cases in more than 4 nights per week. The co-occurrence of polyneuropathy in two of our patients may have influenced the perceived severity of the clinical manifestations of RLS. The existence of RLS symptoms in SLE patients correlated with the impairment of sleep parameters, in contrast with SLE patients without RLS.

\section{Acknowledgements}

This manuscript was partially presented as an e-poster at the 3rd European Academy of Neurology Congress, June 2017, Amsterdam.

\section{Funding}

No funding was received.

\section{Availability of data and materials}

Available upon reasonable request.

\section{Authors' contributions}

All the authors substantially contributed to each of the following aspects of this paper. CFP performed the design and conception of the study, recruited the patients, analyzed and interpreted the data, and drafted the manuscript. AE recruited the patients, analyzed and interpreted the data, and critical revised the manuscript for important intellectually content. LD performed the design and conception of the study, recruited the patients, analyzed and interpreted the data, and critical revised the manuscript for important intellectually content. CF recruited the patients, analyzed and interpreted the data, critical revised the manuscript for important intellectually content. OFP analyzed and interpreted the data, and critical revised the manuscript for important intellectually content. VM analyzed and interpreted the data, and critical revised the manuscript for important intellectually content. SD aided in the literature review, analyzed and interpreted the data, and critical revised the manuscript for important intellectually content. CAS aided in the literature review, analyzed and interpreted the data, and critical revised the manuscript for important intellectually content. All authors read and approved the final manuscript.

\section{Ethics approval and consent to participate}

Approval for the present study was provided by the Ethics Committee of the Transilvania University from Brașov,
Romania. Patient consent was not needed as the database was anonymized.

\section{Patient consent for publication}

Not applicable.

\section{Competing interests}

All the authors declare that they have no competing interests.

\section{Authors' information}

ORCID ID: Cristian Falup-Pecurariu: 0000-0002-7394-7161; Anca Enache: 0000-0002-4647-5939; Liliana Duca: 0000-0002-3062-766X; CamilFotescu:0000-0002-3251-6947; Oana Falup-Pecurariu: 0000-0003-1972-6859; Vlad Monescu: 0000-0002-0506-931X; Stefania Diaconu: 0000-0002-0251-4043; Carmen Adella Sîrbu: 0000-0002-1982-1066.

\section{References}

1. Allen RP, Picchietti D, Hening WA, Trenkwalder C, Walters AS and Montplaisir J, Restless Legs Syndrome Diagnosis and Epidemiology workshop at the National Institutes of Health; International Restless Legs Syndrome Study Group: Restless legs syndrome: Diagnostic criteria, special considerations, and epidemiology. A report from the restless legs syndrome diagnosis and epidemiology workshop at the National Institutes of Health. Sleep Med 4: 101-119, 2003.

2. Allen RP, Picchietti DL, Garcia-Borreguero D, Ondo WG, Walters AS, Winkelman JW, Zucconi M, Ferri R, Trenkwalder $\mathrm{C}$ and Lee HB: International restless legs syndrome study group restless legs syndrome/Willis-Ekbom disease diagnostic criteria: Updated international restless legs syndrome study group (IRLSSG) consensus criteria-history, rationale, description, and significance. Sleep Med 15: 860-873, 2014.

3. Tison F, Crochard A, Léger D, Bouée S, Lainey E and El Hasnaoui A: Epidemiology of restless legs syndrome in French adults: A nationwide survey: The INSTANT Study. Neurology 65: 239-246, 2005.

4. Rothdach AJ, Trenkwalder C, Haberstock J, Keil U and Berger K: Prevalence and risk factors of RLS in an elderly population: The MEMO study. Memory and morbidity in Augsburg elderly. Neurology 54: 1064-1068, 2000.

5. Berger K, Luedemann J, Trenkwalder C, John U and Kessler C: Sex and the risk of restless legs syndrome in the general population. Arch Intern Med 164: 196-202, 2004.

6. Allen RP, Walters AS, Montplaisir J, Hening W, Myers A, Bell TJ and Ferini-Strambi L: Restless legs syndrome prevalence and impact: REST general population study. Arch Intern Med 165: 1286-1292, 2005.

7. Abetz L, Allen R, Follet A, Washburn T, Earley C, Kirsch J and Knight $\mathrm{H}$ : Evaluating the quality of life of patients with restless legs syndrome. Clin Ther 26: 925-935, 2004.

8. Gigli GL, Adorati M, Dolso P, Piani A, Valente M, Brotini S and Budai R: Restless legs syndrome in end-stage renal disease. Sleep Med 5: 309-315, 2004.

9. Chrastina M, Martinkova J, Minar M, Zilinska Z, Valkovic P and Breza J: Impact of kidney transplantation on restless legs syndrome. Bratisl Lek Listy 116: 404-407, 2015.

10. Taylor-Gjevre RM, Gjevre JA, Skomro R and Nair B: Restless legs syndrome in a rheumatoid arthritis patient cohort. J Clin Rheumatol 15: 12-15, 2009.

11. Stehlik R, Arvidsson L and Ulfberg J: Restless legs syndrome is common among female patients with fibromyalgia. Eur Neurol 61: 107-111,2009.

12. Minai OA, Golish JA, Yataco JC, Budev MM, Blazey H and Giannini C: Restless legs syndrome in lung transplant recipients. J Heart Lung Transplant 26: 24-29, 2007. 
13. van Oosterhout WP, van Someren EJ,Louter MA, Schoonman GG, Lammers GJ, Rijsman RM, Ferrari MD and Terwindt GM Restless legs syndrome in migraine patients: Prevalence and severity. Eur J Neurol 23: 1110-1116, 2016.

14. Demirci S, Demirci K, Doğru A, İnal EE, Koyuncuoğlu HR and Şahin M: Restless legs syndrome is associated with poor sleep quality and quality of life in patients with ankylosing spondylitis: A questionnaire-based study. Acta Neurol Belg 116: 329-336, 2016.

15. Sorgun MH, Aksun Z, Atalay YB and Yücesan C: Restless legs syndrome in multiple sclerosis. Turk J Med Sci 45: 1268-1273, 2015.

16. Uglane MT, Westad S and Backe B: Restless legs syndrome in pregnancy is a frequent disorder with a good prognosis. Acta Obstet Gynecol Scand 90: 1046-1048, 2011.

17. Hassan N, Pineau CA, Clarke AE, Vinet E, Ng R and Bernatsky S: Systemic lupus and risk of restless legs syndrome. J Rheumatol 38: 874-876, 2011.
18. Kucuk A, Uslu AU, Yilmaz R, Salbas E, Solak Y and Tunc R: Relationship between prevalence and severity of restless legs syndrome and anemia in patients with systemic lupus erythematosus. Int J Rheum Dis 20: 469-473, 2017.

19. Walters AS, LeBrocq C, Dhar A, Hening W, Rosen R, Allen RP and Trenkwalder C: International restless legs syndrome study group. Validation of the international restless legs syndrome study group rating scale for restless legs syndrome. Sleep Med 4: 121-132, 2003.

20. Abetz L, Vallow SM, Kirsch J, Allen RP, Washburn T and Earley CJ: Validation of the restless legs syndrome quality of life questionnaire. Value Health 8: 157-167, 2005.

21. Buysse DJ, Reynolds CF III, Monk TH, Berman SR and Kupfer DJ: The Pittsburgh sleep quality index: A new instrument for psychiatric practice and research. Psychiatry Res 28: 193-213, 1989.

22. Johns MW: A new method for measuring daytime sleepiness: The Epworth sleepiness scale. Sleep 14: 540-545, 1991. 